\section{Decomposição da diferença da expectativa de vida de Minas Gerais em relação ao Rio de Janeiro e São Paulo, Brasil}

\author{
Disaggregation of differences in life expectancy \\ between the States of Minas Gerais, Rio de \\ Janeiro, and São Paulo, Brazil \\ Análisis sobre la diferencia de expectativa de vida \\ de Minas Gerais, en relación con Río de Janeiro \\ y São Paulo, Brasil
}

\author{
${ }^{1}$ Faculdade de Saúde \\ Pública, Universidade de \\ São Paulo, São Paulo, Brasil. \\ Correspondência \\ A. D. P. Chiavegatto Filho \\ Faculdade de Saúde Pública \\ Universidade de São Paulo. \\ Av. Dr. Arnaldo 715 \\ São Paulo, SP \\ 01246-904, Brasil. \\ alexdiasporto@usp.br
}

\begin{abstract}
Comparisons between States of Brazil are still rare in public health. The States of Minas Gerais, São Paulo, and Rio de Janeiro have adequate mortality registry coverage and are geographically similar, thus making comparison appropriate for identifying possible interventions in public health. Meanwhile, the three States show important socioeconomic differences, consistently worse for Minas Gerais. The study analyzed the disaggregation of differences in life expectancy in Minas Gerais as compared to São Paulo and Rio de Janeiro for 1996, 2000, and 2010, using the Arriaga methodology. Minas Gerais had a higher life expectancy than the two other States for the entire period, in both men and women. Advanced age and chronic illness, especially cancer and ischemic heart disease, were the most important factors in this difference. Differences in lifestyle and physical exercise (better for Minas Gerais than for São Paulo and Rio de Janeiro) could help explain the apparent inconsistency between socioeconomic indicators and life expectancy.
\end{abstract}

Life Expectancy; Chronic Disease; Aged
Alexandre Dias Porto Chiavegatto Filho 1 Ruy Laurenti 1

\section{Resumo}

Ao contrário de outros países, comparações entre regiões brasileiras ainda são pouco realizadas. Minas Gerais, Rio de Janeiro e São Paulo têm boa cobertura do registro de óbitos e semelhanças geográficas, o que permite identificar oportunidades de intervenção em saúde. Por outro lado, existem importantes diferenças socioeconômicas, consistentemente piores em Minas Gerais. Foi realizada uma decomposição da diferença da expectativa de vida de Minas Gerais em relação ao Rio de Janeiro e São Paulo para 1996, 2000 e 2010, utilizando a metodologia de Arriaga. Minas Gerais apresentou maior expectativa de vida em relação aos outros dois estados durante todo o período, tanto para homens quanto para mulheres. O grupo etário dos idosos e as doenças crônicas foram as mais importantes para explicar essa diferença, especialmente neoplasias e doenças isquêmicas do coração. Diferenças no estilo de vida e prática de atividade física (melhores em Minas Gerais do que em relação ao Rio de Janeiro e São Paulo) podem ajudar a explicar a inconsistência entre os níveis socioeconômicos e a situação de saúde.

Expectativa de Vida; Doença Crônica; Idoso 


\section{Introdução}

Comparações da situação de saúde entre os estados têm sido pouco realizadas no Brasil. Isso pode ser explicado pelo fato de a cobertura dos registros de óbitos e nascimentos ter apresentado diferenças importantes segundo regiões até recentemente 1 , o que torna as comparações imprecisas. A cobertura dos óbitos ainda é particularmente ruim para as regiões Norte e Nordeste, onde análises recentes (2010) estimam que 22,4 e $24,5 \%$ dos óbitos, respectivamente, não sejam contabilizados 2 .

A expectativa de vida no Brasil tem aumentado rapidamente nas últimas décadas segundo o Instituto Brasileiro de Geografia e Estatística (IBGE), passando de 70,46 em 2000 para 73,48 em $2010^{3}$. Ao mesmo tempo, tem ocorrido uma melhoria na cobertura do registo de óbitos em todo o país, sobretudo na Região Sudeste, tornando a elaboração de tabelas de vida e o cálculo da expectativa de vida mais adequados 2 .

Minas Gerais, São Paulo e Rio de Janeiro são os três estados mais populosos do Brasil, apresentam renda alta em relação à média brasileira $\mathrm{e}$ fazem fronteira entre si (IBGE. Estados@.http:// www.ibge.gov.br/estadosat/, acessado em 23/ Ago/2012). Apesar das semelhanças, os estados apresentam diferenças significativas na expectativa de vida que não podem ser explicadas por fatores socioeconômicos.

Segundo o Censo Demográfico de 2010, o rendimento nominal mensal de pessoas com mais de 10 anos é consideravelmente menor em Minas Gerais (R\$ 723,16), em relação ao Rio de Janeiro (R\$ 949,29) e São Paulo (R\$ 987,08) (IBGE. Banco de dados agregados. http://www.sidra. ibge.gov.br, acessado em 23/Ago/2012). Resultados semelhantes foram verificados para a educação, utilizando os dados da Pesquisa Nacional por Amostra de Domicílios (PNAD 2008; http:// tabnet.datasus.gov.br/cgi/dh.exe?pnad2008/ pnad.def, acessado em 23/Ago/2012). Em Minas Gerais, $14,5 \%$ dos residentes apresentaram um ano ou menos de escolaridade e $42,28 \%$, oito anos ou mais. No Rio de Janeiro, esses valores foram 10,94\% e 54,07\% e em São Paulo de 10,18\% e 53,87\%. A taxa de alfabetização de pessoas de 10 anos ou mais de idade, segundo o censo demográfico de 2010, foi também pior em Minas Gerais (92,3\%) em relação ao Rio de Janeiro e São Paulo (95,9\% em ambos os casos).

O melhor desempenho socioeconômico do Rio de Janeiro e de São Paulo não se reflete para o caso da expectativa de vida, que tem sido nas últimas décadas maior para Minas Gerais, segundo o IBGE 4. Tal inconsistência entre indicadores socioeconômicos e saúde da população não é frequente em estudos realizados dentro de um mesmo país 5,6,7,8.

O presente estudo tem como objetivo identificar a importância das faixas etárias e das causas básicas de óbito mais frequentes para a maior expectativa de vida verificada para Minas Gerais em relação ao Rio de Janeiro e São Paulo. A semelhança geográfica dos três estados torna a comparação interessante para identificar possibilidades de intervenção em saúde pública.

\section{Metodologia}

\section{Fonte dos dados}

A análise foi realizada para 1996, 2000 e 2010, os anos da última contagem populacional e dos dois últimos censos (Departamento de Informática do SUS. http://www2.datasus.gov.br/DATASUS/ index.php, acessado em 23/Ago/2012). A escolha desses três anos ocorreu porque a utilização de dados originados de projeções de população (períodos entre os censos e contagens) poderia introduzir um viés significativo na análise. Por exemplo, segundo a pesquisa do censo demográfico de 2010, a população residente no Brasil era de 190.755.799 indivíduos. De acordo com os resultados das projeções do IBGE para o ano anterior (2009), a população brasileira teria sido maior (191.481.045), indicando uma diminuição anual de 725 mil residentes, improvável para a atual situação demográfica brasileira.

Os dados de óbitos por faixa etária e causas básicas foram obtidos pelo DATASUS (Departamento de Informática do SUS. http://www2.da tasus.gov.br/DATASUS/index.php, acessado em 23/Ago/2012). Óbitos com idade ignorada foram distribuídos por todas as faixas etárias de forma proporcional aos óbitos com idade determinada. Para menores de um ano de idade, foram utilizados os dados de nascidos vivos do respectivo ano, disponibilizados pelo Sistema de Informações sobre Nascidos Vivos - SINASC (Departamento de Informática do SUS. http://www2.datasus. gov.br/DATASUS/index.php, acessado em 23/ Ago/2012). As causas básicas selecionadas para a análise específica foram as dez mais frequentes para os três estados no ano 2000: neoplasias (código C00 a D48 da Classificação Internacional de Doenças, 10a revisão - CID-10 9), doenças isquêmicas do coração (I20 a I25), doenças cerebrovasculares (I60 a I60), homicídios (X85 a Y09), acidentes (V01 a X59), influenza e pneumonia (J09 a J18), diabetes mellitus (E10 a E14), doenças crônicas das vias aéreas inferiores (J40 a J47), doenças hipertensivas (I10 a I15) e doenças do fígado (K70 a K77). 


\section{Análise estatística}

O cálculo das expectativas de vida para os três estados foi realizado utilizando a metodologia de tábuas de vida de Preston 10. Os valores das médias do número de pessoas-ano vividos em cada intervalo $\left({ }_{n} a_{x}\right)$ para menores de 1 ano de idade $\left({ }_{1} a_{0}\right)$ e para crianças de 1 a 4 anos de idade $\left.{ }_{4} a_{1}\right)$ foram calculados pela utilização das equações de Coale \& Demeny (no caso de ${ }_{1} a_{0}: 0,045+$ $2,684 .{ }_{1} m_{0}$ para homens e $0,053+2,800 \cdot{ }_{1} m_{0}$ para mulheres; no caso de ${ }_{4} a_{1}: 0,413-0,704 \cdot{ }_{1} m_{0}$ para homens e $0,381-0,380{ }_{1} m_{0}$ para mulheres) 11 . Para as outras faixas etárias, foram utilizados os valores das distribuições dos óbitos, assumindo equivalência em relação à função no intervalo de $x-n$ a $x+n$, segundo Preston 10 :

$$
{ }_{n} a_{x}=\frac{\left(-\frac{n}{24}{ }_{n} d_{x-n}+\frac{n}{2}{ }_{n} d_{x}+\frac{n}{24}{ }_{n} d_{x+n}\right)}{{ }_{n} d_{x}}
$$

onde ${ }_{n} d_{x}$ corresponde ao número de pessoas (da coorte inicial padrão de 100 mil indivíduos) que foram a óbito na faixa etária de $x$ a $n$.

Para a comparação da contribuição das faixas etárias e das causas básicas para a diferença entre os três estados, foi utilizada a metodologia de decomposição de Arriaga 12, a mais frequentemente utilizada na literatura $13,14,15$. No caso da decomposição segundo faixa etária, a metodologia permite identificar quantos anos de expectativa de vida são adicionados ou subtraídos de um estado para o outro em razão das diferenças nas taxas de mortalidade conforme idade. A decomposição é feita em três etapas, pelo cálculo sequencial dos efeitos diretos, indiretos e interativos. Por exemplo, no caso da comparação entre São Paulo e Minas Gerais:

${ }_{n} \Delta_{x}=\left[\frac{l_{x}^{S P}}{l_{a}^{S P}}\left(\frac{T_{x}^{M G}-T_{x+i}^{M G}}{l_{x}^{M G}}-\frac{T_{x}^{S P}-T_{x+i}^{S P}}{l_{x}^{S P}}\right)\right]+\left(\frac{T_{x+i}^{M G}}{l_{x+i}^{M G}} \times \frac{\frac{l_{x}^{S P} l_{x+i}^{M G}}{l_{x}^{M G}}-l_{x+i}^{S P}}{l_{a}^{S P}}\right)$

onde $l_{x}$ é o número de pessoas sobrevivendo até a idade $x, l_{a}$ é o número de pessoas iniciais da tabela de vida e $T_{x}$ é o número de pessoas-ano vividos da idade $x$ para cima. O primeiro termo da equação corresponde ao efeito direto, que analisa a diferença no número médio de anos vividos entre $x$ e $x+i$ para os dois estados. Os próximos passos são o cálculo do efeito indireto, que corresponde à diferença de mortalidade dentro da respectiva faixa etária, e o efeito interativo, referente aos sobreviventes de cada faixa etária expostos às diferentes mortalidades entre os dois estados. Os efeitos indiretos e interativos estão agregados na segunda parte da equação. Para a decomposição segundo causas básicas específicas, é utilizada a contribuição de cada causa de óbito para a dife- rença na expectativa de vida em cada faixa etária, considerando-se como proporcional à contribuição dessa causa básica na diferença na taxa de mortalidade total por idades 14 .

\section{Resultados}

A Figura 1 apresenta os resultados da expectativa de vida segundo idades para Minas Gerais, São Paulo e Rio de Janeiro em 1996, 2000 e 2010. Em primeiro lugar, pode-se verificar que a expectativa de vida ao nascer foi sempre maior para mulheres do que para homens nos três períodos, mesmo comparando os piores resultados femininos com os melhores masculinos. Em segundo lugar, a posição relativa dos três estados foi a mesma durante todo o período: Minas Gerais apresentou melhor expectativa de vida ao nascer para ambos os sexos, seguida de São Paulo e do Rio de Janeiro.

A expectativa de vida ao nascer em Minas Gerais para homens aumentou 5,33 anos durante o período, passando de 67,20 em 1996 para 72,53 em 2010. Em relação às mulheres, o aumento foi semelhante (5,44 anos), passando de 74,33 em 1996 para 79,77 em 2010. Em São Paulo, a expectativa de vida dos homens aumentou 6,68 anos no período, de 65,00 a 71,61. Para mulheres, esse aumento foi menor (5,00 anos), passando de 74,27 para 79,27 . Por fim, no Rio de Janeiro a expectativa de vida dos homens aumentou de 6,61, de 62,52 a 69,13 , enquanto para as mulheres o aumentou foi de 5,05 anos, de 72,36 para 77,41.

A Tabela 1 apresenta a contribuição das faixas etárias para a maior expectativa de vida de Minas Gerais em relação a São Paulo e Rio de Janeiro. Em 1996, a faixa etária de menores de um ano foi importante para diminuir a diferença entre os estados, mas esse efeito tem caído ao longo dos anos, por conta da queda da mortalidade infantil em Minas Gerais. Por outro lado, o grupo etário que tem mais contribuído para o aumento da diferença são os idosos (maiores de 65 anos): em 2010, eles foram responsáveis por 1,67 ano da diferença entre a expectativa de vida masculina de Minas Gerais e Rio de Janeiro e 1,16 da diferença entre Minas Gerais e São Paulo. Para as mulheres, essa contribuição foi de 1,33 e 0,73, respectivamente.

Pela Tabela 1 é possível também analisar a variação (de 1996 a 2010) na importância de cada faixa etária para a diferença na expectativa de vida entre os estados. A melhoria da mortalidade infantil em Minas Gerais teve significativo efeito na manutenção de boa parte da diferença em relação aos outros dois estados. Por outro lado, praticamente todas as faixas etárias contribuí- 
Figura 1

Expectativa de vida segundo sexo e faixas etárias para Minas Gerais, São Paulo e Rio de Janeiro, Brasil, 1996, 2000 e 2010.

1a) 1996

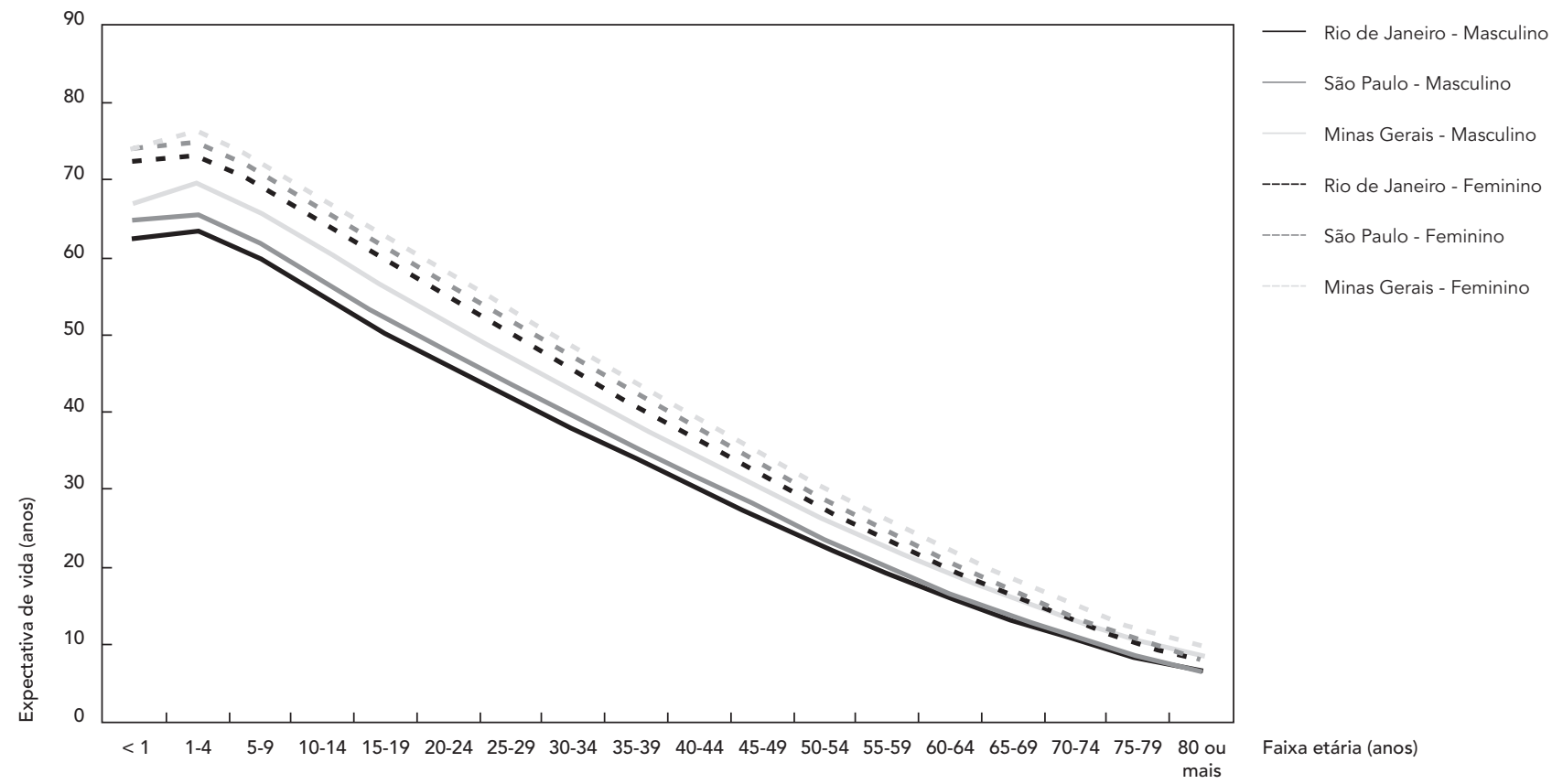

1b) 2000

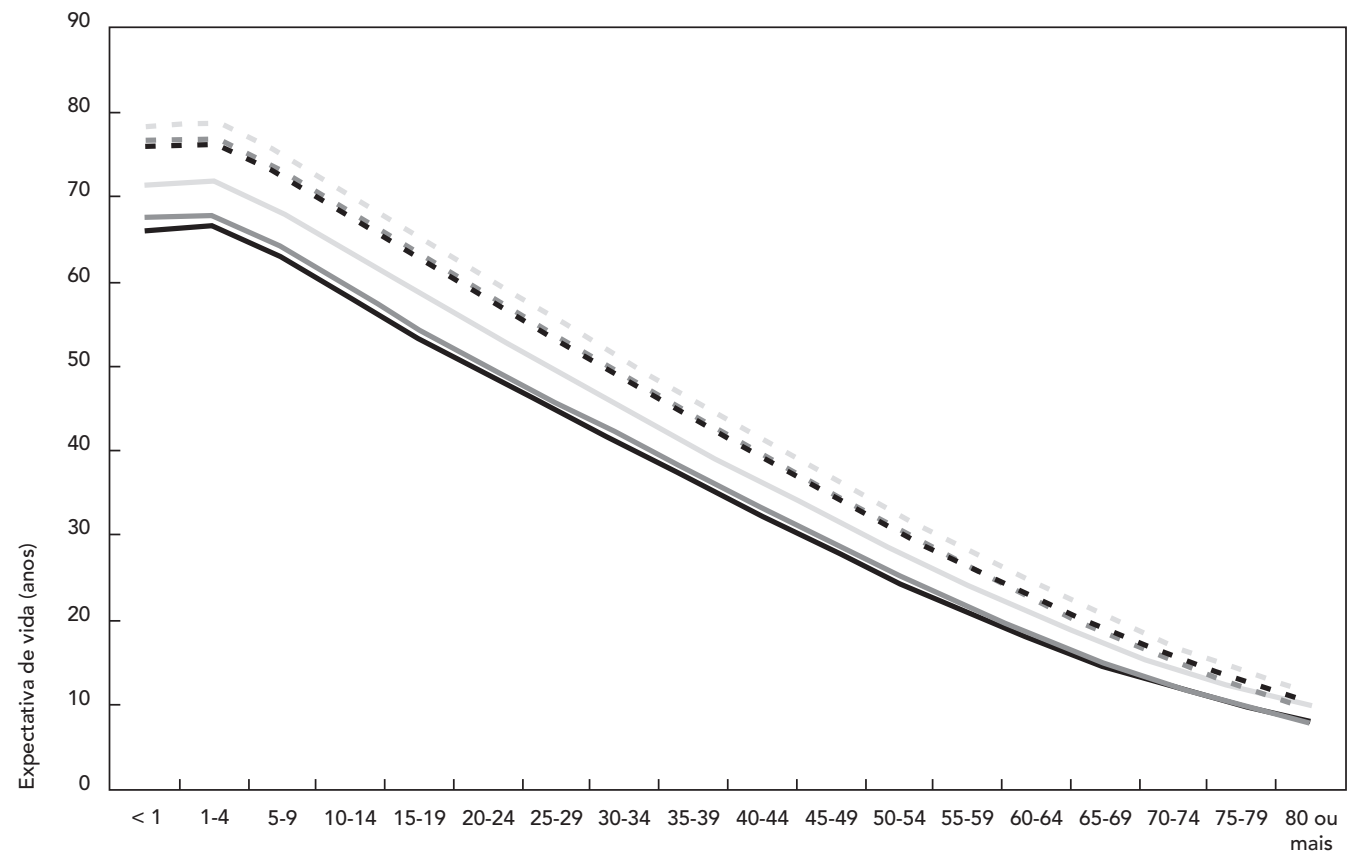

- Rio de Janeiro-Masculino

— São Paulo - Masculino

Minas Gerais - Masculino

Rio de Janeiro - Feminino

São Paulo - Feminino

Minas Gerais - Feminino

(continua) 
1c) 2010

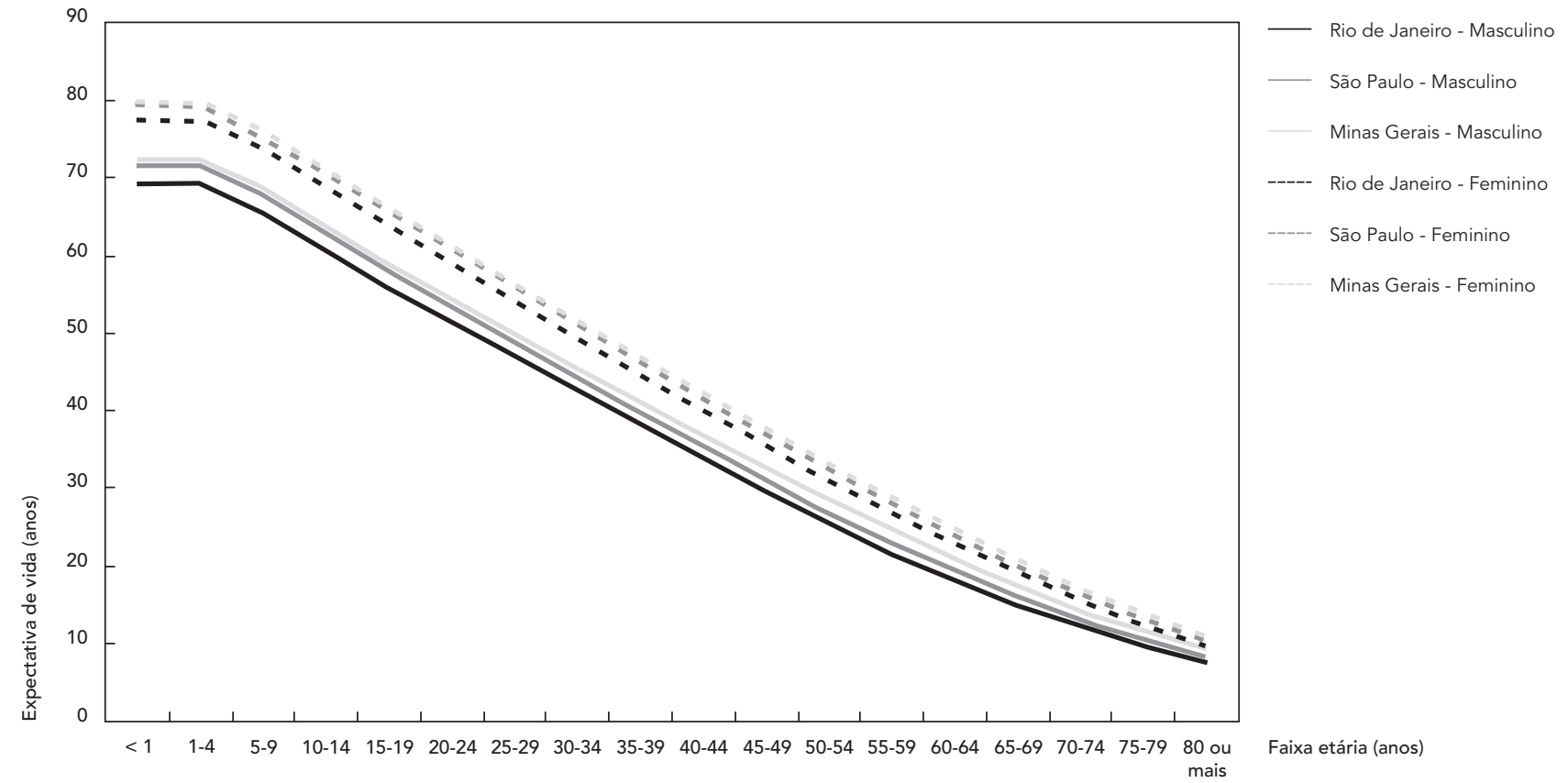

ram para a diminuição da diferença durante o período.

A Tabela 2 apresenta a contribuição das dez causas básica de óbito mais frequentes para a diferença entre as expectativas de vida. Para os homens, a causa básica que mais contribui para a melhor expectativa de vida em Minas Gerais em relação ao Rio de Janeiro durante todo período foi homicídio. Em relação a São Paulo, em 2010 o homicídio passa a ser responsável pela diminuição da diferença da expectativa de vida em relação a Minas Gerais. Isso se deve ao fato de a taxa de mortalidade por homicídio ter caído para São Paulo, enquanto para Minas Gerais houve aumento. A diferença total entre Minas Gerais e os outros dois estados foi também bastante influenciada pelos óbitos por doença isquêmica do coração.

Em relação às mulheres, as doenças isquêmicas do coração e as neoplasias foram as causas básicas mais responsáveis pela maior expectativa de vida de Minas Gerais em relação a São Paulo e ao Rio de Janeiro. Influenza e pneumonia também tiveram importância para essa diferença, especialmente em 2010. Ao contrário dos homens, a baixa mortalidade por homicídio entre as mu- lheres pouco explicou a diferença na expectativa de vida. Foram verificadas, entretanto, algumas diferenças entre os estados, como no caso do diabetes, que foi menos importante para explicar a diferença entre Minas Gerais e São Paulo do que no caso do Rio de Janeiro. É importante mencionar que para ambos os sexos, a maioria das causas básicas teve efeito no aumento da diferença entre a expectativa de vida em Minas Gerais em relação aos outros dois estados.

Pela Tabela 2 é possível também identificar as mudanças na importância das causas básicas para a diferença entre as expectativas de vida durante o período. Tanto para homens quanto para mulheres, a maioria das causas contribuiu para a diminuição da diferença entre os estados durante o período, particularmente o homicídio para os homens. Em relação ao total das dez causas básicas mais frequentes, elas foram responsáveis pela diminuição da diferença entre os estados durante o período, especialmente entre os homens. Nesse caso, em 1996 eram responsáveis por 5,01 anos da diferença entre a expectativa de vida masculina de Minas Gerais e Rio de Janeiro, enquanto em 2010 esse valor diminuiu para 2,60. No caso da diferença entre Minas 
Tabela 1

Contribuição das faixas etárias para a diferença da expectativa de vida entre Minas Gerais (MG) em relação ao Rio de Janeiro (RJ) e São Paulo (SP), Brasil,

1996,2000 e 2010.

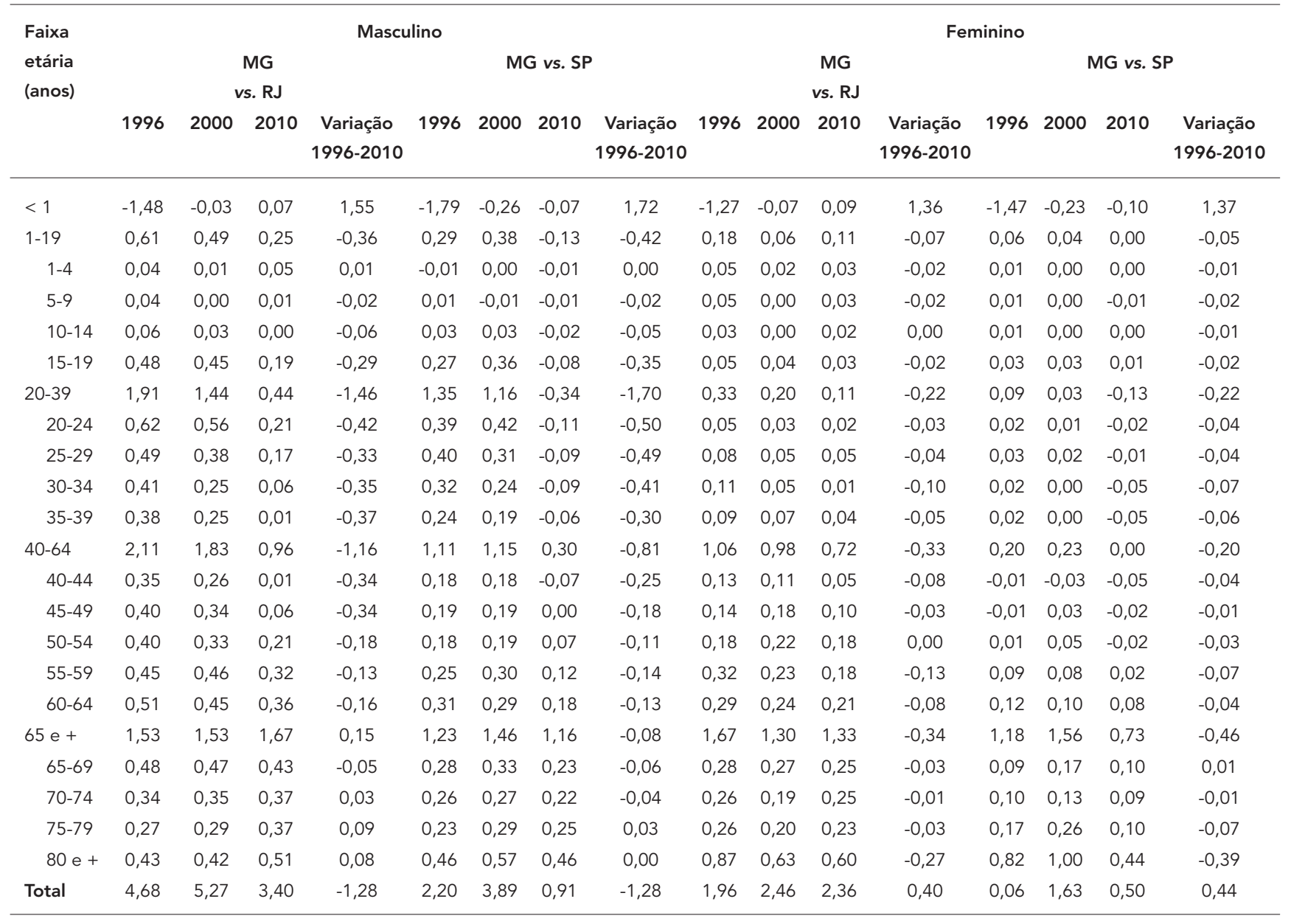

Gerais e São Paulo, os valores foram de 3,89 e 1,38 , respectivamente.

\section{Discussão}

O estudo permitiu realizar a decomposição da diferença na expectativa de vida entre Minas Gerais em relação a São Paulo e ao Rio de Janeiro. O Estado de Minas Gerais apresentou maior expectativa de vida em relação ao Rio de Janeiro e São Paulo durante todo o período, tanto para homens quanto para mulheres.

Em 2010, a expectativa de vida de Minas Gerais foi maior em 0,91 e 3,40 anos em relação a São Paulo e Rio de Janeiro para homens e 0,50 e 2,36 para mulheres. Em relação aos homens, os homicídios foram responsáveis por 0,82 ano da diferença entre Minas Gerais e o Rio de Janeiro, enquanto que para Minas Gerais e São Paulo as doenças isquêmicas do coração contribuíram para 0,62 ano da diferença. Analisando as mulheres, a causa básica mais importante para essa diferença foram as neoplasias para o Rio de Janeiro $(0,45$ anos) e novamente as doenças isquêmicas do coração para São Paulo $(0,56)$. Os casos de influenza e pneumonia, que explicaram pouco dessa diferença em 1996 e 2000, passaram a ser importante em 2010, contribuindo, no caso do Rio de Janeiro, para 0,24 ano da diferença entre homens e 0,25 para mulheres, e, no caso de São Paulo, para 0,35 e 0,32 ano, respectivamente.

Em relação às faixas etárias, em 2010 o grupo dos idosos (com mais de 65 anos) foi o que mais contribuiu para a maior expectativa de vida em Minas Gerais em relação a São Paulo (1,16 e 0,73, para homens e mulheres, respectivamente) e Rio de Janeiro $(1,67$ e 1,33). A mortalidade in- 
Tabela 2

Contribuição das causas básicas mais frequentes para a diferença da expectativa de vida entre Minas Gerais (MG) em relação ao Rio de Janeiro (RJ) e São Paulo (SP), Brasil, 1996, 2000 e 2010.

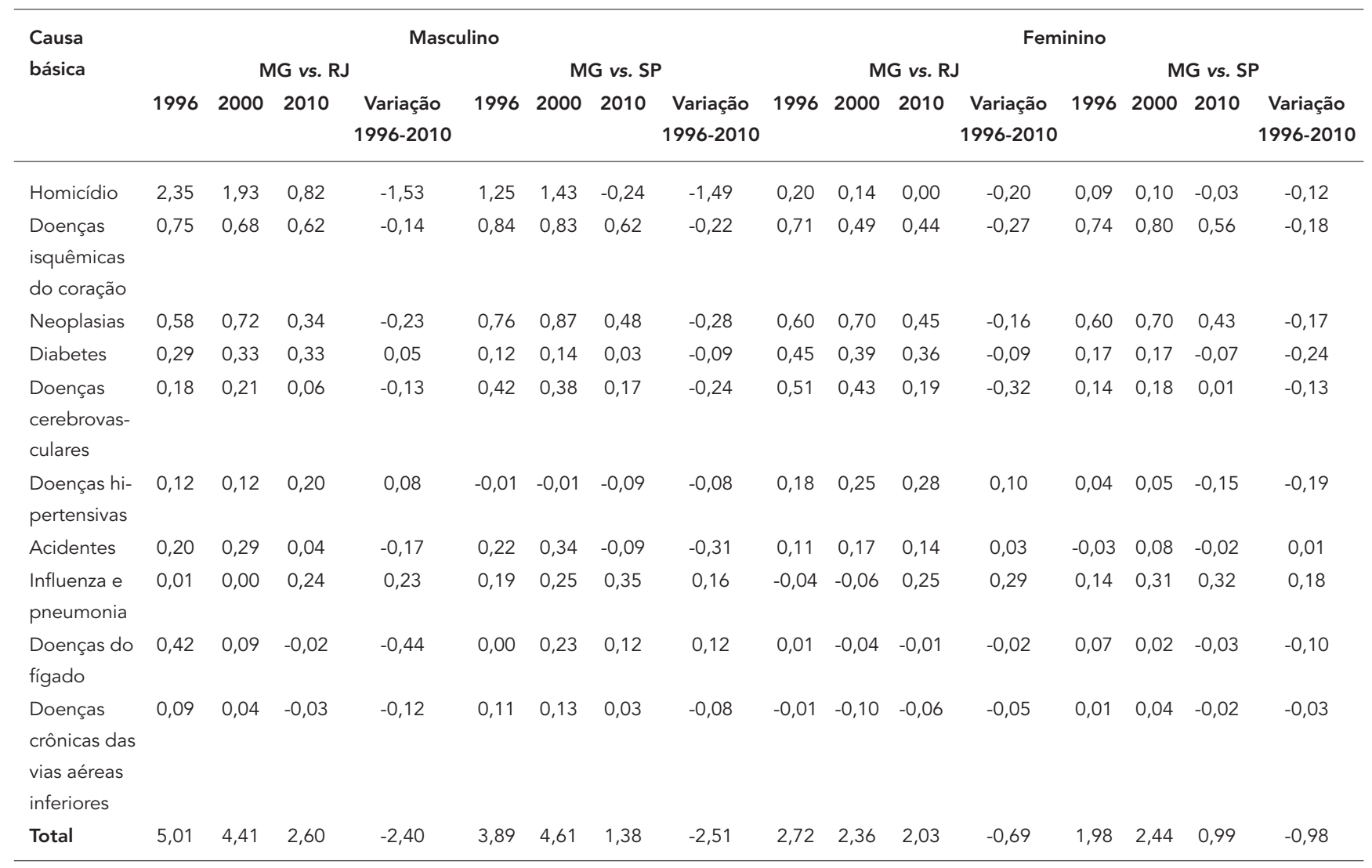

fantil, que era importante para a diminuição da diferença entre a expectativa de vida de Minas Gerais e os outros dois estados em 1996, deixou de ser importante em 2010 (explicando menos de 0,1 ano para ambos os estados e sexos). Isso se deveu, em particular, à rápida melhoria da queda da mortalidade infantil em Minas Gerais, que passou de 48,58 por mil nascidos vivos em 1996 para 13,08 em 2010.

A diferença na expectativa de vida entre Minas Gerais e os dois outros estados caiu durante o período analisado. A causa que mais contribui para essa diminuição entre os homens foi o homicídio. Essa mudança aconteceu por dois motivos. Em primeiro lugar, a taxa de mortalidade por homicídios aumentou em Minas Gerais de 13,2 homicídios para 100 mil habitantes em 1996 para 33,53 em 2010. Além disso, no mesmo período, esses valores diminuíram em São Paulo de 67,61 para 25,71, e de 114,23 para 64,24 no Rio de Janeiro. Assim, o homicídio tornou-se menos relevante para explicar a diferença, apesar de continuar importante no caso do Rio de Janeiro.
A importância da maioria das doenças crônicas para o aumento da diferença entre Minas Gerais e os outros dois estados abre algumas oportunidades para intervenções na área da saúde. Analisando 2010, as doenças isquêmicas do coração e as neoplasias foram as causas mais importantes para explicar a melhor expectativa de vida em Minas Gerais.

Inconsistências entre renda e situação de saúde são mais frequentes em estudos que compararam países. Nesses casos, a diferença pode ser resultado da distribuição de fatores de risco 16 . Um estudo recente, que analisou a expectativa de vida entre países europeus, apontou para a importância de fatores como diferenças alimentares e tabagismo para explicar divergências entre renda e situação de saúde 17 . No caso dos Estados Unidos, estima-se que o aumento da prevalência de obesidade leve a uma diminuição da expectativa de vida em 1,02 ano nos próximos 15 anos 18.

Assim como nesses estudos, as diferenças de estilo de vida e fatores de risco entre Minas Ge- 
rais, São Paulo e Rio de Janeiro podem ter tido efeito importante. Segundo a PNAD 2008 (http:// tabnet.datasus.gov.br/cgi/dh.exe?pnad2008/ pnad.def, acessado em 23/Ago/2012), 23,8\% dos residentes do Rio de Janeiro e 23,2\% dos de São Paulo são fisicamente inativos, percentual maior do que para Minas Gerais (17,5\%). Um estudo das capitais brasileiras realizado por meios de inquéritos telefônicos em 2006 verificou que a prática de atividade física regular foi maior em Belo Horizonte em relação ao Rio de Janeiro e São Paulo 19. A prevalência de sobrepeso e obesidade também é diferente entre as três capitais. Uma análise de inquéritos domiciliares constatou que em Belo Horizonte 40,7\% dos adultos apresentaram excesso de peso, enquanto no Rio de Janeiro e São Paulo esse valor foi maior, de 49,2\% e 44,8\%, respectivamente 20 .

O presente estudo tem como limitação a existência de diferenças na cobertura dos óbitos entre Minas Gerais, São Paulo e Rio de Janeiro. Apesar de em 2000 e 2010 a cobertura ter sido semelhante à dos países desenvolvidos para os três estados 2,21, em 1990 a cobertura dos óbitos em Minas Gerais foi considerada apenas como "satisfatória" 1, enquanto para o Rio de Janeiro e São Paulo a cobertura foi "boa" (não foi feita a análise para 1996, mas a melhoria gradual da cobertura sugere um valor intermediário). Logo, parte da mudança da diferença na expectativa de vida no período pode ser atribuída à melhoria da cobertura em Minas Gerais. Por outro lado, espera-se que em 2010 a diferença na expectativa de vida reflita diferenças reais na saúde da população.

A identificação da real importância das causas básicas para as diferenças na expectativa de vida pode também ter sido prejudicada pela presença de óbitos por causas mal definidas (capítulo XVIII da CID-10). Apesar da pequena diminui- ção durante o período $(8,79 \%$ do total dos óbitos nos três estados em 1996 e 7,36\% em 2010), pode ter aumentado a proporção de mal definidas para algumas doenças enquanto a identificação da verdadeira causa básica pode ter melhorado para outras 22 .

Outra limitação é o uso de decomposição segundo causas básicas, que não permite a análise multidimensional da saúde. Ou seja, os mecanismos de interação entre as diversas doenças não são incluídos. Por exemplo, casos de óbitos por hipertensão são frequentemente codificados como doença isquêmica do coração 23 , em razão das regras de seleção da CID-10, o que subestima a importância relativa das doenças hipertensivas.

\section{Conclusão}

As diferenças existentes nos índices socioeconômicos entre os três estados (melhores para São Paulo e Rio de Janeiro) não se refletem em relação à expectativa de vida (melhor para Minas Gerais). O presente estudo verificou que a diferença na expectativa de vida é influenciada por quase todas as faixas etárias (sobretudo no caso de idosos) e causas básicas mais frequentes (no caso de 2010, especialmente as doenças isquêmicas do coração e neoplasias), uma possível indicação de melhores indicadores de saúde como um todo. A análise realizada pelo estudo, no entanto, não possibilitou identificar mecanismos causais que possam explicar a inconsistência entre os índices socioeconômicos e a expectativa de vida. Estudos futuros, utilizando dados individuais de sobrevivência, e controlando por características do estilo de vida, podem ajudar a identificar os motivos dessa diferença. 


\section{Resumen}

Al contrario que en otros países, todavía se realizan pocas comparaciones entre las diferentes regiones brasileñas. Minas Gerais, Río de Janeiro y São Paulo tienen una buena cobertura del registro de óbitos y semejanzas geográficas, lo que permite identificar oportunidades de intervención en salud. Por otro lado, existen importantes diferencias socioeconómicas, perceptiblemente peores en Minas Gerais. Se realizó un análisis sobre la diferencia de expectativa de vida de Minas Gerais, en relación con Río de Janeiro y São Paulo durante los años 1996, 2000 y 2010, utilizando la metodología de Arriaga. Minas Gerais presentó una mayor expectativa de vida en relación a los otros dos estados durante todo el período, tanto para hombres, como para mujeres. El grupo etario de los ancianos y las enfermedades crónicas fueron los más importantes para explicar esa diferencia, principalmente neoplasias y enfermedades isquémicas del corazón. Diferencias en el estilo de vida y práctica de actividad física (mejores en Minas Gerais en comparación con Río de Janeiro y São Paulo) pueden ayudar a explicar la inconsistencia entre los niveles socioeconómicos y la situación de salud.

Esperanza de Vida; Enfermedad Crónica; Anciano

\section{Colaboradores}

A. D. P. Chiavegatto Filho contribuiu com a análise e interpretação dos dados, redação do artigo e aprovação da versão final. R. Laurenti contribuiu com a concepção, análise e interpretação dos dados, revisão crítica do artigo e aprovação da versão final.

\section{Agradecimentos}

Coordenação de Aperfeiçoamento de Pessoal de Nível Superior (Capes; processo: 0692-11-5).

\section{Referências}

1. Paes NA. Avaliação da cobertura dos registros de óbitos dos estados brasileiros em 2000. Rev Saúde Pública 2005; 39:882-90.

2. Instituto Brasileiro de Geografia e Estatística. Estatísticas do registro civil, 2010. Rio de Janeiro: Instituto Brasileiro de Geografia e Estatística; 2011.

3. Instituto Brasileiro de Geografia e Estatística. Brasil: tábua completa de mortalidade, 2010. Rio de Janeiro: Instituto Brasileiro de Geografia e Estatística; 2011.
4. Instituto Brasileiro de Geografia e Estatística. Síntese de indicadores sociais: uma análise das condições de vida da população brasileira. Rio de Janeiro: Instituto Brasileiro de Geografia e Estatística; 2010

5. Jia H, Lubetkin EI. The statewide burden of obesity, smoking, low income and chronic diseases in the United States. J Public Health 2009; 31:496-505.

6. Auger N, Alix C, Zang G, Daniel M. Sex, age, deprivation and patterns in life expectancy in Quebec Canada: a population-based study. BMC Public Health 2010; 10:161 
7. Subramanian S, Kawachi I, Kennedy BP. Does the state you live in make a difference? Multilevel analysis of self-rated health in the US. Soc Sci Med 2001; 53:9-19.

8. Santos SM, Chor D, Werneck GL, Coutinho ESF. Associação entre fatores contextuais e autoavaliação de saúde: uma revisão sistemática de estudos multinível Association between contextual factors and self-rated health: a systematic review. Cad Saúde Pública 2007; 23:2533-54.

9. Organização Mundial da Saúde. Classificação estatística internacional de doenças e problemas relacionados à saúde. 10a Rev. São Paulo: Edusp; 2003.

10. Preston SH, Heuveline P, Guillot M. Demography: measuring and modeling population processes Oxford: Wiley; 2001.

11. Coale AJ, Demeny P. Regional model life tables and stable population. New York: Academic Press 1983.

12. Arriaga EE. Measuring and explaining the change in life expectancies. Demography 1984; 21:83-96.

13. Auger N, Harper S, Barry AD, Trempe N, Daniel M. Life expectancy gap between the Francophone majority and Anglophone minority of a Canadian population. Eur J Epidemiol 2011; 27:27-38.

14. Harper S, Lynch J, Burris S, Smith GD. Trends in the black-white life expectancy gap in the United States, 1983-2003. JAMA 2007; 297:1224-32.

15. Yang S, Khang YH, Harper S, Davey Smith G, Leon DA, Lynch J. Understanding the rapid increase in life expectancy in South Korea. Am J Public Health 2010; 100:896-903.
16. Lopez AD, Mathers CD, Ezzati M, Jamison DT, Murray CJL. Global and regional burden of disease and risk factors, 2001: systematic analysis of population health data. Lancet 2006; 367:1747-57.

17. Leon DA. Trends in European life expectancy: a salutary view. Int J Epidemiol 2011; 40:271-7.

18. Stewart ST, Cutler DM, Rosen AB. Forecasting the effects of obesity and smoking on US life expectancy. New Engl J Med 2009; 361:2252-60.

19. Malta DC, Moura EC, Castro AM, Cruz DKA, Morais Neto OL, Monteiro CA. Padrão de atividade física em adultos brasileiros: resultados de um inquérito por entrevistas telefônicas, 2006. Epidemiol Serv Saúde 2009; 18:7-16.

20. Instituto Nacional do Câncer. Inquérito domiciliar sobre comportamentos de risco e morbidade referida de doenças e agravos não transmissíveis. http://www.inca.gov.br/inquerito/ (acessado em 23/Ago/2012).

21. World Health Organization. Causes of death 2008: data sources and methods. Geneva: World Health Organization; 2011.

22. Jorge MHP, Laurenti R, Lima-Costa MF, Gotlieb SLD. A mortalidade de idosos no Brasil: a questão das causas mal definidas. Epidemiol Serv Saúde 2008; 17:271-81.

23. Chiavegatto Filho ADP, Laurenti R, Gotlieb SLD, Mello Jorge M. Mortalidade por doença hipertensiva em mulheres de 20 a 49 anos no Município de São Paulo, SP, Brasil. Rev Bras Epidemiol 2004; 7:252-8.

Recebido em 20/Fev/2012

Versão final reapresentada em 04/Jan/2013

Aprovado em 31/Jan/2013 